\title{
Detecção de cor da pele na melhoria do redimensionamento de imagem baseado em conteúdo
}

\author{
Savio Lopes Rabelo, Tamara Arruda Pereira, Francisco Nivando Bezerra \\ Instituto Federal de Educação, Ciência e Tecnologia do Ceará (IFCE), \\ Eixo Tecnológico de Computação, Campus Maracanaú - CE - Brasil \\ \{saviorabelo.ti, tamaraarrudap\}@gmail.com, nivando@ifce.edu.br
}

\begin{abstract}
Seam Carving is a content-aware resizing method capable of modifying the width or height of pictures. This algorithm applies an energy function to evaluate the importance of each pixel. In special cases, such as images that contain people, the method frequently presents deformation of objects, due the energy function not being able to detect a person. In this context, this paper presents a modification of the energy function used in seam carving aiming to better preserve people in images. This energy function is generated from a neural network that has as input arguments the color of the skin in order to classify the pixel in skin or non-skin.
\end{abstract}

Resumo. O Seam Carving é um método de redimensionamento capaz de modificar a largura ou altura de imagens sendo sensivel ao seu conteúdo, esse algoritmo aplica uma função de energia para avaliar a importância de cada pixel. Em casos particulares, como imagens que contém pessoas, o método apresenta frequente deformação de objetos, devido a função de energia não ser apta a detecção de pessoa. Com isso em vista, este artigo apresenta uma formulação na função de energia para o Seam Carving específica para a preservação de pessoas nas imagens. Esta função de energia é elaborada a partir de uma rede neural que tem como argumentos de entrada a cor da pele para classificar o pixel em pele ou não pele.

\section{Introdução}

Redimensionar uma imagem é um recurso frequentemente utilizado em operações de processamento de imagens. A habilidade de manter objetos importantes em uma imagem após o seu redimensionamento é característica de um método eficiente. Técnicas tradicionais como o dimensionamento em que o tamanho da imagem pode ser alterado usando interpolação, preserva os elementos visuais, mas causa distorção perceptível se a proporção for diferente entre a entrada e saída. Outra forma é cortar a imagem de entrada, mas esse método pode remover dados de interesse se a resolução de saída for significativamente menor que a de entrada [Senturk \& Akgun 2017].

O Seam Carving é um método de redimensionamento que se preocupa com a preservação do conteúdo da imagem. Ele utiliza uma função de energia para avaliar a importância de cada pixel. Entropia, métodos baseados em gradientes, mapas de saliência, segmentação, decomposição via transformada de wavelet, são alguns métodos conhecidos na literatura que podem ser utilizados como função de energia [Oliveira 2016]. Esse método possui a desvantagem de frequentes deformações dos objetos da imagem. 
Um caso específico de distorção de conteúdo utilizando o Seam Carving pode ser visto em redimensionamento aplicado a imagens com pessoas. A tarefa de detecção de objetos importantes em uma imagem possui adversidades que tornam o problema desafiador. Para a detecção de pessoas, a natureza não rígida do corpo humano produz inúmeras poses possíveis, além da variação do tamanho devido à posição da câmera, fundos desordenados podem causar a camuflagem do objeto e oclusões podem ocorrer em imagens que têm várias pessoas interagindo [Nguyen et al. 2016]. A cor da pele é uma característica que possui a vantagem de não variar em relação a rotação, tamanho e oclusões parciais. O método apresentado neste artigo utiliza a cor da pele como argumento de entrada para uma rede neural a fim de classificar os pixels em pele ou não pele, e usa essa classificação na função de energia do Seam Carving com o intuito de preservar pessoas após o redimensionamento.

Este artigo está organizado da seguinte maneira: a Seção 2 descreve o método Seam Carving; a Seção 3 descreve a detecção de pele; a Seção 4 apresenta o método proposto nesse trabalho: Redimensionamento com preservação de pessoas; a Seção 5 explica como os experimentos para validação da proposta foram realizados, bem como, os resultados obtidos; e por fim, a conclusão é apresentada na Seção 6.

\section{Seam Carving}

Um método de redimensionamento eficaz modifica o tamanho (altura ou largura) de uma imagem e deve ser capaz de preservar o conteúdo importante sempre que possível. O Seam Carving [Avidan \& Shamir 2007] é um algoritmo de redimensionamento de imagens que utiliza uma função de energia para determinar o conteúdo relevante que deve ser preservado [Dong et al. 2009].

O algoritmo insere ou remove um conjunto de pixels conectados verticalmente ou horizontalmente. Para redução do tamanho da imagem preservando a sua estrutura, removemos mais pixels de baixa energia e menos pixels de alta energia [Avidan \& Shamir 2007].

A Figura 1 mostra o Seam Carving aplicado a uma imagem que contém uma pessoa. Para o redimensionamento, foram retirados $20 \%$ de seams do tamanho total da imagem. Pode ser visto na Figura 1(c) que a imagem sofreu deformação após a remoção dos seams, isso acontece quando a função de energia não é adequada para o problema.

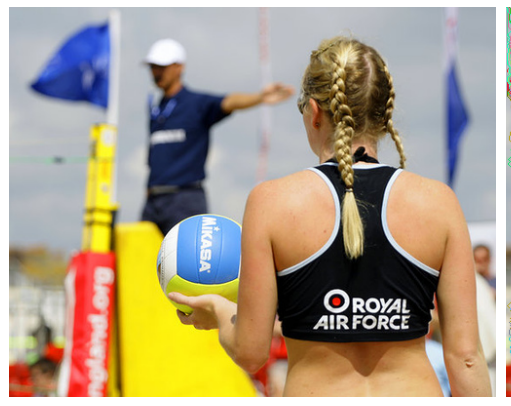

(a)

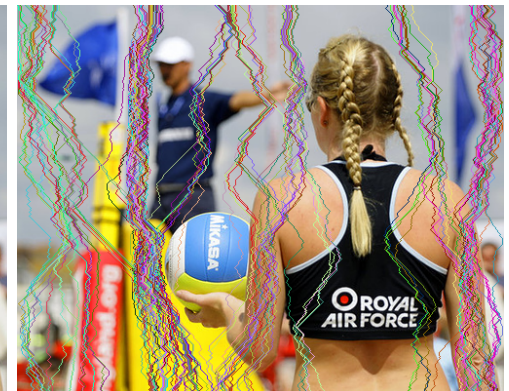

(b)

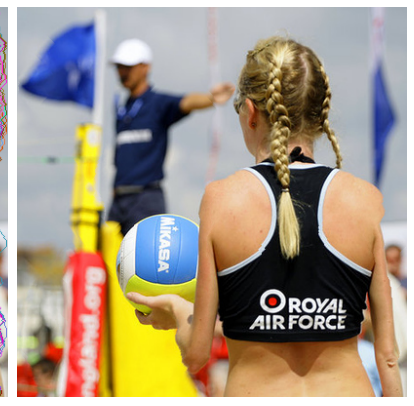

(c)

Figura 1. Uso do Seam Carving. (a) Imagem original [Rubinstein et al. 2010]. (b) $20 \%$ dos seams vertical escolhidos para serem removidos. (c) Redução de $20 \%$ (100 seams) da largura. 


\section{Detecção de Pele}

A segmentação de imagem é o processo de dividir uma imagem em regiões não sobrepostas que são grupos de pixels homogêneos conectados [Liu \& Chung 2011]. A detecção de pele nada mais é que uma segmentação que divide os pixels da imagem em dois grupos: região de pele (qualquer parte exposta do corpo humano que aparece na imagem, como rostos, ombros, mãos, pernas) e não pele, levando em consideração suas características que podem ser cor ou textura, por exemplo.

O processo de detecção de pele é utilizado em diversos problemas de processamento de imagem, como detecção de rosto [Zhipeng et al. 2010], reconhecimento facial [Kang et al. 2016], análise de gestos [Han et al. 2009], recuperação de imagens baseada em conteúdo [Bhute \& Meshram 2014], detecção de pessoas nuas [Perez et al. 2017], entre outros.

A cor da pele é uma propriedade eficiente, robusta e invariante a alterações, como rotação, escala e oclusões parciais [Kakumanu et al. 2007], mas a detecção de pele humana que utiliza a cor como premissa em imagens complexas provou ser um problema desafiador porque a cor da pele pode variar drasticamente em sua aparência devido aos fatores: iluminação, raça, envelhecimento, condições de imagem e fundo complexo [Yuetao \& Nana 2011].

O método de detecção de pele baseado em pixel classifica em duas classes: pele ou não pele, individualmente, sem considerar seus vizinhos. O detector de pele procura pixels com cores que correspondem ao modelo de cor da pele [Phung et al. 2003]. Há métodos que tentam criar detectores de pele com a capacidade de aprender com um conjunto de dados de treinamento sem criar um modelo explícito da cor da pele [Naji et al. 2018], como é o caso das redes neurais artificiais, que é empregado neste artigo. A saída da rede neural para classificação dos pixels é representada na Equação 1:

$$
D P(x, y)= \begin{cases}1, & \text { se } \mathbf{I}(x, y) \in \text { cor pele } \\ 0, & \text { caso contrário }\end{cases}
$$

onde I é a imagem de entrada da rede e $\mathbf{I}(x, y)$ é o pixel na posição $(x, y)$.

\section{Redimensionamento com Preservação de Pessoas}

Este trabalho apresenta uma nova função de energia para o Seam Carving que privilegia a preservação de pessoas. O Redimensionamento com Preservação de Pessoas (RPP) combina o algoritmo do Seam Carving com uma rede neural capaz de classificar pixel em pele e não pele. Para isso, a função de energia do Seam Carving é alterada e o valor $+\infty$ é atribuído para todos os pixels classificados como pele e os demais seguem as mesmas diretrizes de [Avidan \& Shamir 2007] ao utilizar na função de energia a magnitude do gradiente, definido como um filtro baseado em derivada que realça os detalhes e detecta bordas.

Para cada pixel da imagem I, é calculado o valor da magnitude do vetor gradiente naquele pixel somando os módulos das derivadas na direção vertical e horizontal como é mostrado na Equação 2: 


$$
e(\mathbf{I})=\left|\frac{\partial}{\partial x} \mathbf{I}\right|+\left|\frac{\partial}{\partial y} \mathbf{I}\right| .
$$

A função de energia $e$ do Seam Carving é substituída pela função $e_{D P}$ que atribui $+\infty$ aos pixels classificados por $D P(x, y)=1$ e em caso contrário, é atribuído a função de energia original, como é mostrado na Equação 3:

$$
e_{D P}(x, y)= \begin{cases}+\infty, & \text { se } D P(x, y)=1 \\ e(x, y), & \text { caso contrário. }\end{cases}
$$

O uso das funções de energia é mostrado na Figura 2. A Figura 2(a) é a imagem original; a Figura 2(b) é o resultado depois de aplicar a função de energia $e$ do Seam Carving, quanto mais clara a região, mais energia e mais relevante; a Figura 2(c) é a classificação do pixel usando a função $D P$; e na Figura 2(d) mostra a imagem após o uso da função de energia $e_{D P}$ do RPP, no qual os pixels classificados como pele estão mais evidentes comparados à Figura 2(b).

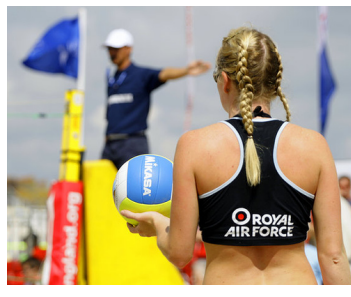

(a)

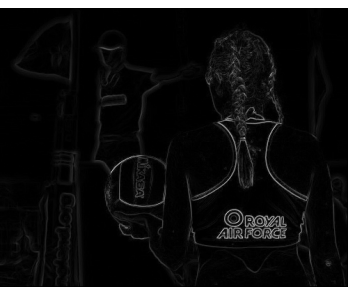

(b)

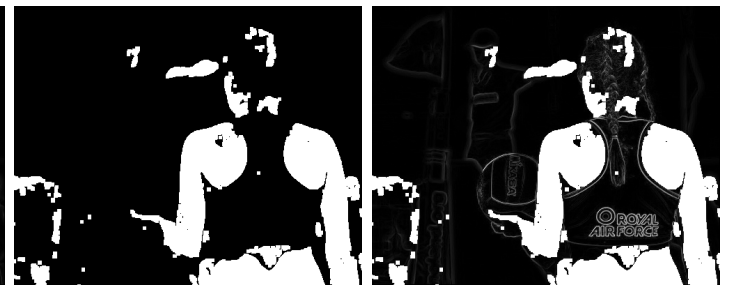

(c) (d)

Figura 2. Funções de energia. (a) Imagem original [Rubinstein et al. 2010]. (b) Função $e$. (c) Função $D P$. (d) Função $e_{D P}$.

A Figura 3 mostra as etapas do RPP. Primeiramente, realiza-se um préprocessamento na base de dados de treinamento para retirada de ambiguidade, em seguida, esses dados são passados como parâmetro para o treinamento da rede. Depois que a rede foi treinada e avaliada, são repassadas as imagens de teste do Seam Carving, e então, são feitas as detecções de pele nessas imagens. Em seguida, realiza-se uma operação morfológica utilizando um fechamento, para retirada de pequenas estruturas. Por fim, aplica-se a função de energia $e_{D P}$ e os seams de menos importância são retirados, resultando nas imagens redimensionadas. 


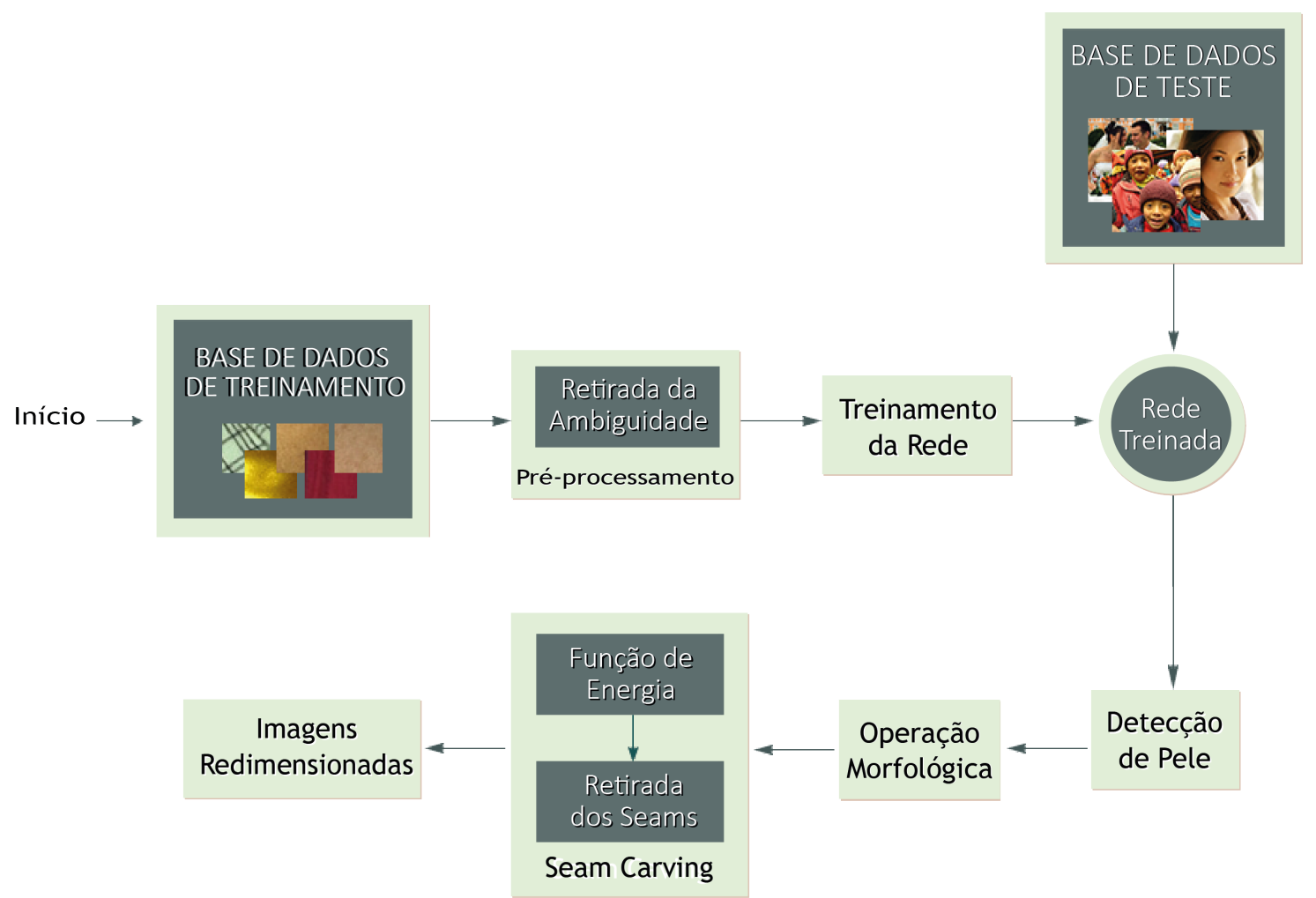

Figura 3. Etapas para redimensionamento com preservação de pessoas.

\section{Experimentos}

Nesta Seção são descritos os experimentos e os resultados alcançados pelo método proposto neste artigo. Para todos os experimentos, foi utilizado um computador com a seguinte configuração: processador Intel(R) Core(TM) i7-6500U a $2.5 \mathrm{GHz}$ com 8 GB de RAM e executando Windows 10. Além disso, foi usado o MATLAB (desenvolvido por MathWorks) para implementação.

\subsection{Experimentos para detecção de pele}

Para a classificação dos pixels em pele e não pele, a rede neural escolhida foi o Perceptron Multicamada (do inglês, Multilayer Perceptron, MLP) [Rumelhart et al. 1988]. A escolha da base de dados para treinamento da rede neural é importante para amenizar problemas como raça e envelhecimento. A base de dados de imagens de pele humana SFA [Casati et al. 2013] foi escolhida por ser um conjunto de dados que contém uma coleção de imagens diversificada. A SFA foi construída com base em imagens faciais das bases de dados FERET [Phillips et al. 1998] e AR [M. Martinez \& Benavente 1998]. A SFA possui 3354 amostras de pele e 5590 amostras de não pele.

Os sistemas de cores RGB, HSV e YCbCr foram utilizados como atributos da camada de entrada do MLP para classificação da pele, mesma abordagem utilizada em [Hajiarbabi \& Agah 2015]. Todos os dados são pré-processados para retirada de ambiguidades. Os pixels que tinham os mesmos valores entre pele e não pele foram atribuídos à categoria de pele. 
Como a cor da pele sofre variações com a iluminação, algumas abordagens como a de [Yuetao \& Nana 2011, Baskan et al. 2002] assumem que os componentes de crominância da cor do tom de pele são independentes do componente de luminância. Portanto, o canal de iluminação é desprezado e um espaço de cores bidimensional é escolhido, em vez de um espaço de cores tridimensional. Dessa forma, o HS pode substituir o espaço de cores do HSV [Baskan et al. 2002] e o YCbCr pode ser substituído por $\mathrm{CbCr}$ [Habili et al. 2004], descartando a luminância desse modelos. Em [Moallem et al. 2011, Naji 2013], os autores assumem que tais abordagens mostram baixo desempenho quando aplicadas em imagens complexas devido à perda de algumas informações de cor quando um pixel colorido é expresso em espaço bidimensional, em vez de tridimensional. O simples fato de ignorar qualquer informação de cor afeta a precisão do sistema. Com objetivo de comparação, este trabalho aplica as duas técnicas, tanto a utilização do espaço de cor tridimensional quanto o bidimensional.

Cerca de $60 \%$ das amostras foram utilizadas para treinamento, $20 \%$ para validação e $20 \%$ para teste. O MLP foi treinado com diferentes números de neurônios na camada oculta, variando entre 6 e 40.

Para a avaliação dos resultados alcançados na classificação dos pixels em pele e não pele, foram utilizados as seguintes métricas: a precisão ou valor preditivo positivo (VPP), taxa de sensibilidade ou taxa positiva verdadeira (TPV), especificidade ou taxa real negativa (SPC) e acurácia (ACC). As Equações são apresentadas a seguir:

$$
\begin{gathered}
V P P=\frac{V P}{V P+F P}, \\
T P V=\frac{V P}{V P+V N}, \\
S P C=\frac{V N}{N}=\frac{V N}{F P+V N}, \\
A C C=\frac{V P+V N}{P+N},
\end{gathered}
$$

onde $\mathrm{P}$ é o número de pixels de pele e $\mathrm{N}$ é o número de pixels de não pele. VP é o número de pixels de pele classificados corretamente. VN é o número de pixels que não são de pele corretamente classificados. FP é o número de pixels que não são da pele, mas que foram classificados incorretamente como pixels da pele. FN é o número de pixels de pele classificados incorretamente como pixels não pele.

A Figura 4 mostra como a rede se comporta com diferentes números de neurônios da camada oculta do MLP. É possível ver no gráfico que a rede que obteve a melhor acurácia no conjunto de validação possui 34 neurônios na camada oculta, ela foi a rede utilizada nos teste apresentados nesta Seção. 


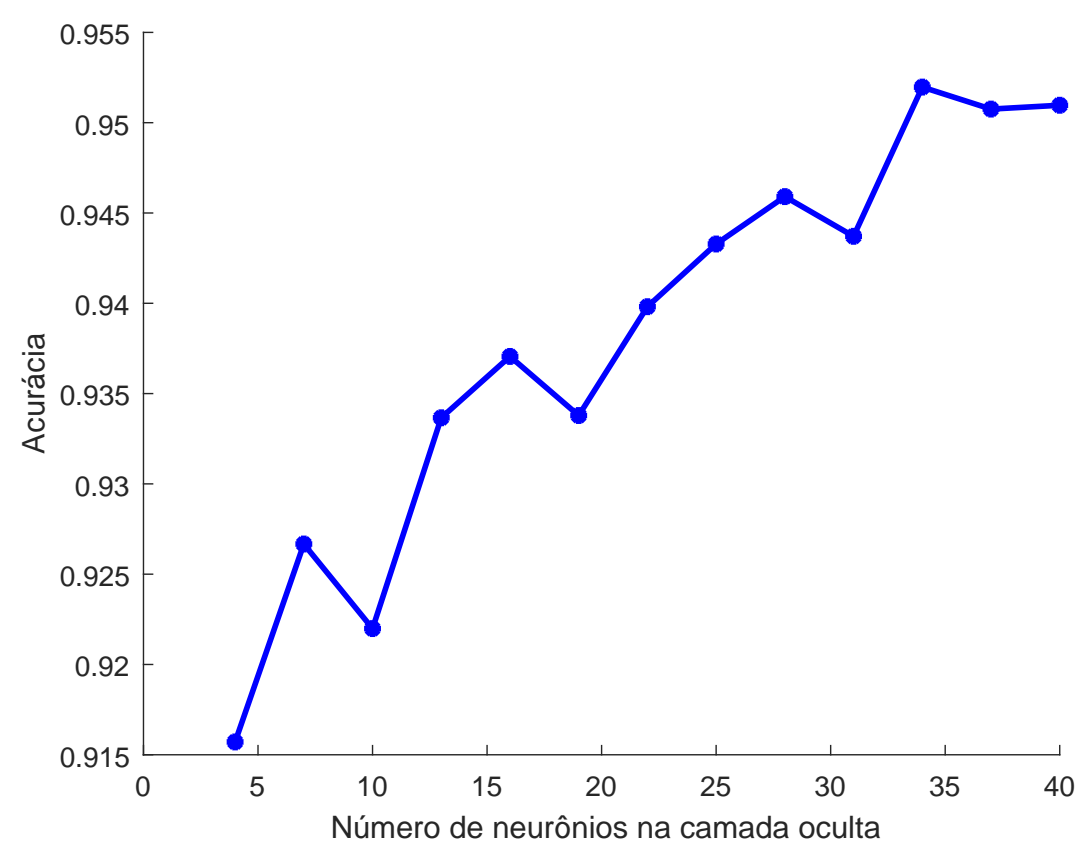

Figura 4. Evolução da Acurácia com a variação do número de neurônios na camada oculta da rede neural.

Foram testadas separadamente 7 entradas para o MLP: RGB, HSV, HS, YCbCr, $\mathrm{CbCr}$, RGBHSVYCbCr e RGBHSCbCr. As redes foram comparadas com as métricas de avaliação e os resultados são apresentados na Tabela 1.

Tabela 1. Teste de redes neurais com diferentes modelos de cor.

\begin{tabular}{ccccc}
\hline \multirow{2}{*}{ Redes } & \multicolumn{4}{c}{ Métricas } \\
\cline { 2 - 5 } & VPP & TPV & SPC & ACC \\
\hline RGB & 96,20 & 94,12 & 96,32 & 95,20 \\
HSV & 96,57 & 93,07 & 96,44 & 94,69 \\
HS & 93,30 & 91,15 & 93,14 & 92,12 \\
YCbCr & 96,65 & 93,37 & 96,53 & 94,90 \\
CbCr & 93,41 & 88,18 & 92,99 & 90,44 \\
RGBHSVYCbCr & 96,64 & $\mathbf{9 5 , 1 2}$ & 96,59 & $\mathbf{9 5 , 8 5}$ \\
RGBHSCbCr & $\mathbf{9 6 , 6 8}$ & 94,87 & $\mathbf{9 6 , 6 1}$ & 95,73 \\
\hline
\end{tabular}

Como pode ser observado na Tabela 1, a abordagem de desprezar o canal de iluminação dos sistemas de cores $\mathrm{HSV}$ e YCbCr se mostrou ineficiente, pois as redes com entradas $\mathrm{HSV}$ e $\mathrm{YCbCr}$ apresentaram resultados superiores às redes $\mathrm{HS}$ e $\mathrm{CbCr}$, respectivamente, para todas as métricas. A rede com a entrada RGBHSVYCbCr apresentou melhor acurácia entre todas as outras redes, inclusive à $\mathrm{RGBHSCbCr}$, esse resultado é compreensível ao analisar separadamente os resultados das redes RGB, HSV e YCbCr, que se mostraram mais eficientes que HS e $\mathrm{CbCr}$. A rede com melhor resultado de acurácia foi utilizada na Seção 5.2 para os experimentos do RPP. 
Os modelos RGB, RGBHSVYCbCr e RGBHSCbCr apresentaram individualmente os melhores resultados, então foram combinados com métodos de reforço para obter um único classificador de cor da pele. Três métodos de reforço foram aplicados: $A N D, O R$ e VOTING. As saídas das três diferentes redes neurais (RGB, HSV e YCbCr) foram utilizadas. Se a saída mostrar que o pixel pertence à pele humana, ela é considerada 1 , caso contrário, 0 . No método $A N D$, todas as saídas devem confirmar que o pixel pertence à pele humana. No método $O R$, se apenas uma saída mostra que o pixel pertence à pele humana, então isso é suficiente para a decisão final de considerar esse pixel como pele humana. No método VOTING, pelo menos duas saídas de três devem mostrar que o pixel pertence à pele humana, de modo que a decisão final considera esse pixel como pele humana. Os resultados alcançados utilizando essa metodologia são apresentados na Tabela 2.

Tabela 2. Resultados para os métodos de reforço AND, OR e VOTING no SFA.

\begin{tabular}{ccccc}
\hline \multirow{2}{*}{ Métodos de Reforço } & \multicolumn{4}{c}{ Métricas } \\
\cline { 2 - 5 } & VPP & TPV & SPC & ACC \\
\hline AND & $\mathbf{9 8 , 0 2}$ & 93,10 & $\mathbf{9 7 , 9 1}$ & 95,38 \\
OR & 94,97 & $\mathbf{9 6 , 2 4}$ & 95,03 & 95,63 \\
VOTING & 96,84 & 95,01 & 96,78 & $\mathbf{9 5 , 8 8}$ \\
\hline
\end{tabular}

A operação $A N D$ tem mais alta precisão e especificidade em comparação com os outros dois métodos; no entanto, ele teve uma sensibilidade muito menor, o que significa que muitos pixels da pele são considerados pixels não pele. Em termos de sensibilidade, o método $O R$ reconhece muito mais pixels da pele corretamente em comparação com os outros dois métodos. O método VOTING tem a maior precisão entre esses três métodos.

\subsection{Experimentos para o redimensionamento com preservação de pessoas}

Para todos os experimentos apresentados nessa Seção, foram utilizadas duas bases de dados para teste, a RetargetMe [Rubinstein et al. 2010] que é composta por 80 imagens e a base de dados NRID [Fang et al. 2012] que contém 35 imagens. As duas base de dados são diversificadas e amplamente utilizadas em problemas de redimensionamento e abrangem imagens de pessoas e paisagens. O método proposto foi aplicado somente à imagens de pessoas.

Nas Figuras 5, 6 e 7 são mostrados os redimensionamentos com 20\% e $30 \%$ do total da largura das imagens, utilizando os métodos Seam Carving e RPP. Nas Figuras 5 e 6, o método RPP apresentou resultados visivelmente superiores ao Seam Carving preservando as áreas que foram classificadas como pele. Já o método Seam Carving, apresentou distorção de informação relevante das pessoas presentes na imagem. 


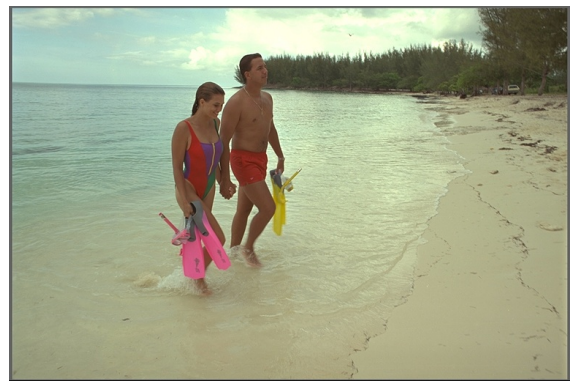

(a)

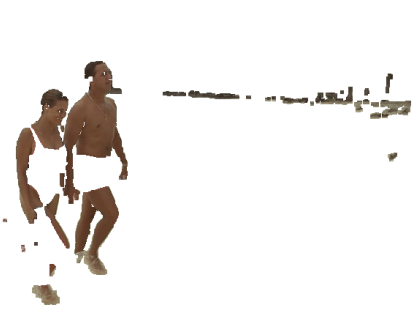

(d)

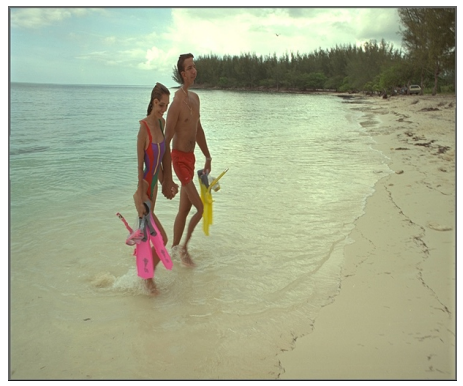

(b)

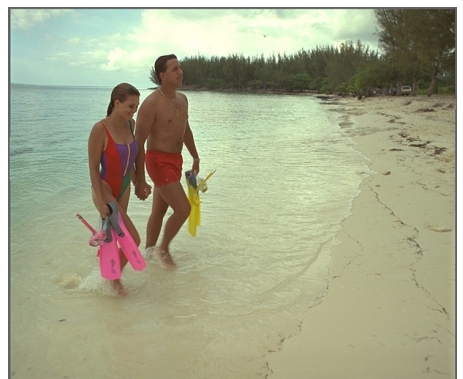

(e)

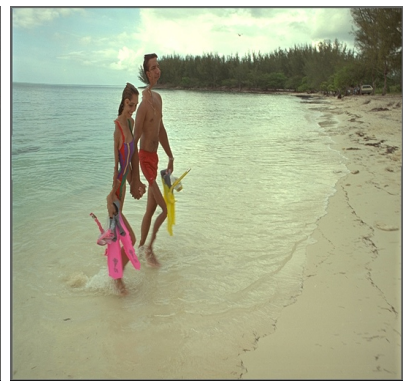

(c)

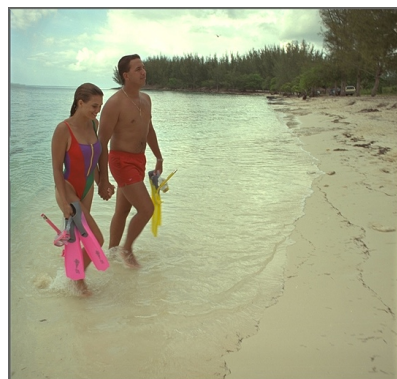

(f)

Figura 5. Resultado do Seam Carving e do RPP. (a) Imagem original extraída da base NRID [Fang et al. 2012]. (b) Redução de 20\% (154 seams) da largura pelo Seam Carving. (c) Redução de 30\% (230 seams) da largura pelo Seam Carving. (d) Detecção de pele aplicada a imagem original. (e) Redução de $20 \%$ (154 seams) da largura pelo RPP. (f) Redução de 30\% (230 seams) da largura pelo RPP.

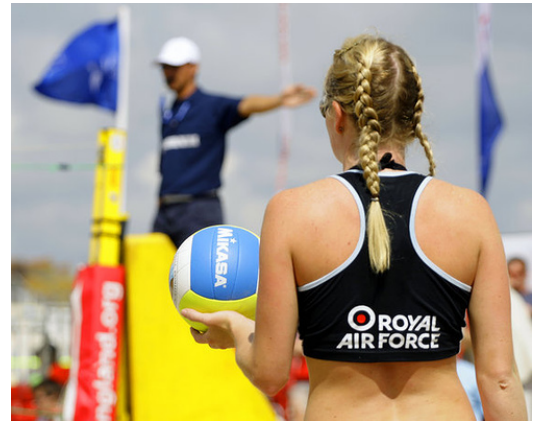

(a)

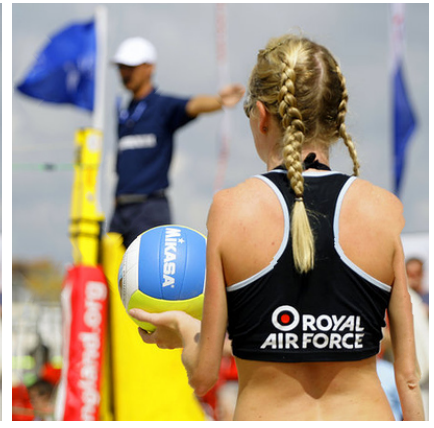

(b)

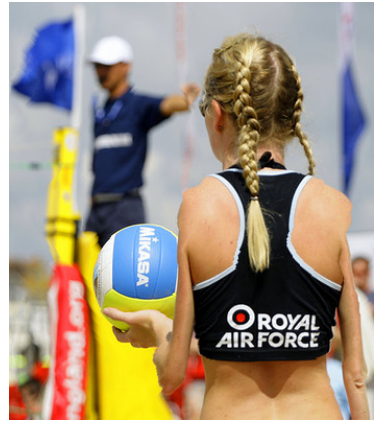

(c)

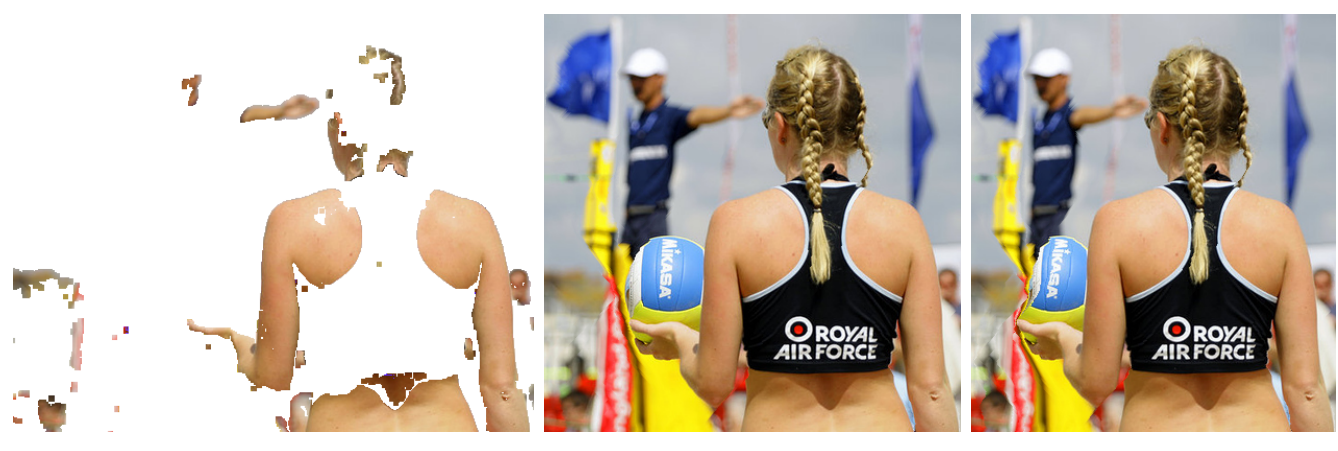

(d)

(e)

(f)

Figura 6. Resultado do Seam Carving e do RPP. (a) Imagem original extraída da base RetargetMe [Rubinstein et al. 2010]. (b) Redução de 20\% (100 seams) da largura pelo Seam Carving. (c) Redução de 30\% (150 seams) da largura pelo Seam Carving. (d) Detecção de pele aplicada a imagem original. (e) Redução de $20 \%$ (100 seams) da largura pelo RPP. (f) Redução de 30\% (150 seams) da largura pelo RPP. 
Na Figura 7, o método RPP mostrou resultados superiores ao Seam Carving, por se tratar de uma imagem com fundo complexo que possui cores muito parecidas com o conjunto de cores da pele. Como pode ser visto na Figura 7(d), a distorção do corpo das pessoas utilizando o RPP é visível (perceptíveis na cabeça e cabelo das duas pessoas e no braço do homem), embora isso tenha acontecido, as áreas que foram classificadas como pele sofreram poucas distorções.

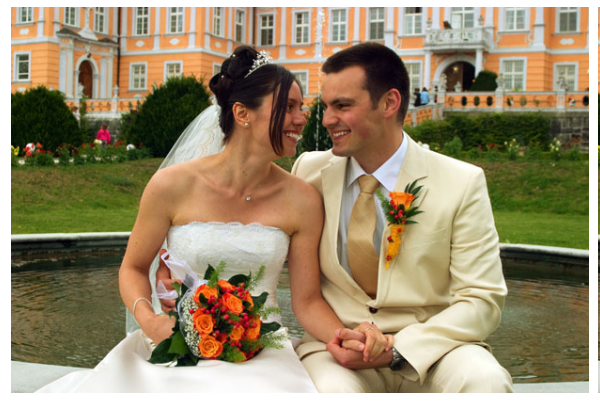

(a)

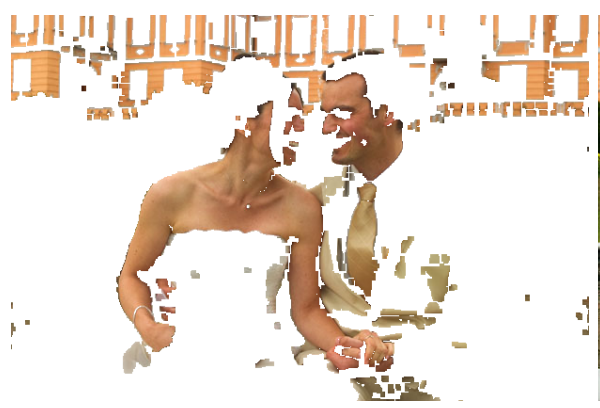

(d)

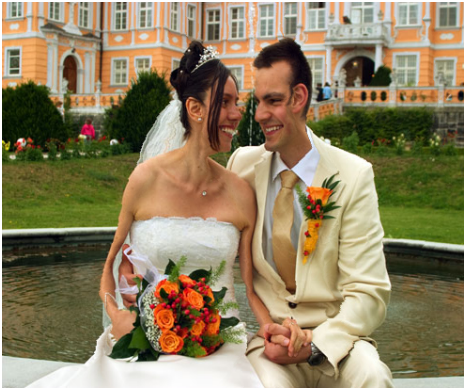

(b)

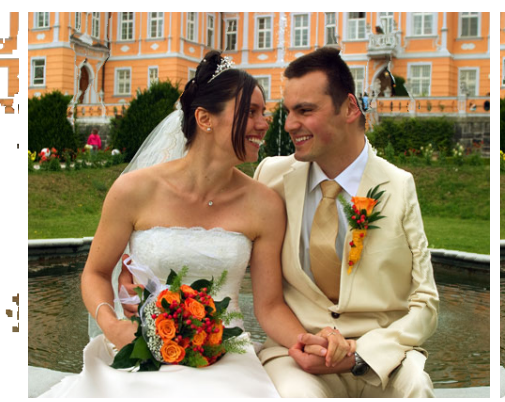

(e)

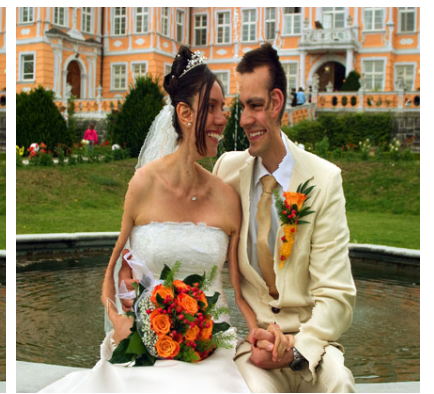

(c)

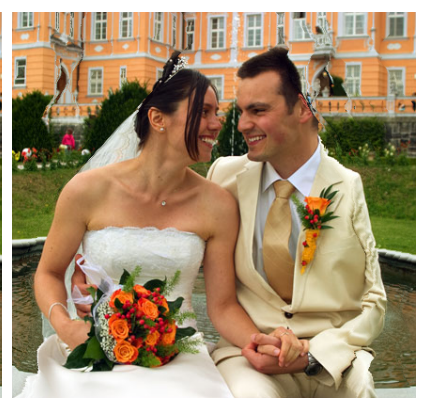

(f)

Figura 7. Resultado do Seam Carving e do RPP. (a) Imagem original extraída da base RetargetMe [Rubinstein et al. 2010]. (b) Redução de 20\% (123 seams) da largura pelo Seam Carving. (c) Redução de 30\% (185 seams) da largura pelo Seam Carving. (d) Detecção de pele aplicada a imagem original. (e) Redução de $20 \%$ (123 seams) da largura pelo RPP. Redução de 30\% (185 seams) da largura pelo RPP.

\section{Conclusão}

Apresentamos neste artigo o método RPP que emprega uma nova função de energia para o Seam Carving elaborada a partir de uma rede neural capaz de detectar cor de pele. A rede foi treinada utilizando separadamente 7 entradas usando diferentes modelos de cor que, em seguida, foram avaliadas levando em conta a precisão, sensibilidade, especificidade e acurácia, com objetivo de encontrar a melhor entrada para classificar pixels em pele ou não pele. Duas das entradas testadas utilizava a técnica de descartar o canal de iluminação para evitar problemas com a variação de iluminação, mas se mostraram ineficientes.

Os melhores resultados de acurácia foram obtidos com os modelos de cor RGB, RGBHSVYCbCr e RGBHSCbCr. Esses modelos de representação foram escolhidos como entrada para 3 redes neurais cujos resultados foram combinados por métodos de reforço, $A N D, O R$ e VOTING, para obter um único classificador de cor da pele. 
A acurácia e a precisão que verifica a validade e a confiabilidade do modelo assumiram valores significativos, consequentemente, a rede consegue detectar a pele corretamente na maioria dos casos. Os resultados apresentados demonstraram a viabilidade da proposta, uma vez que o RPP mostrou desempenho no mínimo equivalente aquele alcançado através do Seam Carving. As distorções perceptíveis causadas com a utilização do método RPP são minimizadas se comparadas com às obtidas pelo Seam Carving.

Como trabalhos futuros, pode-se sugerir a proposição de métricas de avaliação qualitativa dos resultados para melhor comparação com outros métodos, especialmente com o Seam Carving convencional. Isso permitirá uma melhor decisão sobre as entradas da rede neural, permitindo, por exemplo, considerar também métodos de detecção de pele baseados em região e em texturas.

\section{Referências}

Avidan, S. \& Shamir, A. (2007). Seam carving for content-aware image resizing. 26(3):10.

Baskan, S., Bulut, M. M., \& Atalay, V. (2002). Projection based method for segmentation of human face and its evaluation. Pattern Recognition Letters, 23(14):1623-1629.

Bhute, A. N. \& Meshram, B. (2014). Content based image indexing and retrieval. arXiv preprint arXiv:1401.1742.

Casati, J. P. B., Moraes, D. R., \& Rodrigues, E. L. L. (2013). Sfa: A human skin image database based on feret and ar facial images. In IX workshop de Visao Computational, Rio de Janeiro.

Dong, W., Zhou, N., Paul, J.-C., \& Zhang, X. (2009). Optimized image resizing using seam carving and scaling. In ACM Transactions on Graphics (TOG), volume 28, page 125. ACM.

Fang, Y., Chen, Z., Lin, W., \& Lin, C.-W. (2012). Saliency detection in the compressed domain for adaptive image retargeting. IEEE Transactions on Image Processing, 21(9):3888-3901.

Habili, N., Lim, C. C., \& Moini, A. (2004). Segmentation of the face and hands in sign language video sequences using color and motion cues. IEEE Transactions on Circuits and Systems for Video Technology, 14(8):1086-1097.

Hajiarbabi, M. \& Agah, A. (2015). Human skin color detection using neural networks. Journal of Intelligent Systems, 24(4):425-436.

Han, J., Awad, G., \& Sutherland, A. (2009). Automatic skin segmentation and tracking in sign language recognition. IET Computer Vision, 3(1):24-35.

Kakumanu, P., Makrogiannis, S., \& Bourbakis, N. (2007). A survey of skin-color modeling and detection methods. Pattern recognition, 40(3):1106-1122.

Kang, S., Choi, B., \& Jo, D. (2016). Faces detection method based on skin color modeling. Journal of Systems Architecture, 64:100-109.

Liu, C.-C. \& Chung, P.-C. (2011). Objects extraction algorithm of color image using adaptive forecasting filters created automatically. International Journal of Innovative Computing, Information and Control, 7(10):5771-5787. 
M. Martinez, A. \& Benavente, R. (1998). The ar face database. Tech. Rep. 24 CVC Technical Report.

Moallem, P., Mousavi, B. S., \& Monadjemi, S. A. (2011). A novel fuzzy rule base system for pose independent faces detection. Applied Soft Computing, 11(2):1801-1810.

Naji, S., Jalab, H. A., \& Kareem, S. A. (2018). A survey on skin detection in colored images. Artificial Intelligence Review.

Naji, S. A. (2013). Human face detection from colour images based on multi-skin models, rule-based geometrical knowledge, and artificial neural network. PhD thesis, University of Malaya.

Nguyen, D. T., Li, W., \& Ogunbona, P. O. (2016). Human detection from images and videos: A survey. Pattern Recognition, 51:148-175.

Oliveira, S. A. F. D. (2016). Uma abordagem com base em algoritmos genéticos para o redimensionamento de imagens sensível ao conteúdo. Dissertação, Instituto Federal de Educação, Ciência e Tecnologia - Campus Fortaleza.

Perez, M., Avila, S., Moreira, D., Moraes, D., Testoni, V., Valle, E., Goldenstein, S., \& Rocha, A. (2017). Video pornography detection through deep learning techniques and motion information. Neurocomputing, 230:279-293.

Phillips, P. J., Wechsler, H., Huang, J., \& Rauss, P. J. (1998). The feret database and evaluation procedure for face-recognition algorithms. Image and vision computing, 16(5):295-306.

Phung, S. L., Bouzerdoum, A., \& Chai, D. (2003). Skin segmentation using color and edge information. In Seventh International Symposium on Signal Processing and Its Applications, 2003. Proceedings., volume 1, pages 525-528. IEEE.

Rubinstein, M., Gutierrez, D., Sorkine, O., \& Shamir, A. (2010). A comparative study of image retargeting. ACM Transactions on Graphics (Proc. SIGGRAPH Asia), 29(6):160:1-160:10.

Rumelhart, D. E., Hinton, G. E., Williams, R. J., et al. (1988). Learning representations by back-propagating errors. Cognitive modeling, 5(3):1.

Senturk, Z. K. \& Akgun, D. (2017). Seam carving based image resizing detection using hybrid features. Tehnicki Vjesnik-Technical Gazette, 24(6):1825-1833.

Yuetao, D. \& Nana, Y. (2011). Research of face detection in color image based on skin color. Energy Procedia, 13:9395-9401.

Zhipeng, C., Junda, H., \& Wenbin, Z. (2010). Face detection system based on skin color model. In 2010 International Conference on Networking and Digital Society, volume 2, pages 664-667. IEEE. 